\title{
Disseminated Juvenile Xanthogranuloma
}

National Cancer Institute

\section{Source}

National Cancer Institute. Disseminated Juvenile Xanthogranuloma. NCI Thesaurus. Code C81772.

Juvenile xanthogranuloma disseminated to extracutaneous sites including mucosal surfaces, lung, central nervous system, pituitary, lymph nodes, eye, liver, and bone marrow. It is characterized by the presence of lipid-laden, foamy histiocytes and Toutontype giant cells forming nodules in the affected anatomic sites. 